\title{
Perícia Psicológica no Abuso Sexual de Crianças e Adolescentes
}

\author{
Luiziana Souto Schaefer ${ }^{1}$ \\ Silvana Rossetto \\ Christian Haag Kristensen \\ Pontificia Universidade Católica do Rio Grande do Sul
}

\begin{abstract}
RESUMO - Ao mesmo tempo em que denúncias de abuso sexual contra crianças e adolescentes são frequentes, implicando em medidas protetivas urgentes para as vítimas e punições para os perpetradores, também são crescentes os casos de falsas denúncias. Este artigo tem como principal objetivo revisar, com base na literatura nacional e internacional recente, o papel da perícia psicológica no abuso sexual infantojuvenil. Na comum inexistência de vestígios físicos, uma avaliação psicológica abrangente demonstra-se imprescindível, devendo integrar diferentes fontes de informação e indicadores, já que alguns destes são contraditórios e inespecíficos. O perito deve possuir formação na área de atuação e conhecimentos sobre a legislação vigente, além de assegurar que a avaliação não se torne um elemento abusivo para o periciado.
\end{abstract}

Palavras-chave: violência contra crianças e adolescentes; abuso sexual; perícia psicológica.

\section{Forensic Psychological Examination in Child and Adolescent Sexual Abuse}

\begin{abstract}
At the same time that allegations of sexual abuse against children and adolescents are frequent, enhancing urgent protective measures for victims and punishment for the perpetrators, there is also an increasing frequency of void allegations. This paper aims to review, from recent national and international literature, the role of psychological examination in child and adolescent sexual abuse. The literature review shows that since lack of physical evidence is common, a wide psychological evaluation is essential, and must integrate different information sources and indicators, some of which are inconsistent and nonspecific. An expert must have extensive training in the field and knowledge of the current legislation, making sure that the evaluation will not turn out to be traumatic for the client.
\end{abstract}

Keywords: violence against children and adolescents; sexual abuse; psychological expertise.

Nos casos de abuso sexual contra crianças e adolescentes, têm sido verificados sérios prejuízos ao desenvolvimento infantojuvenil, com repercussões cognitivas, emocionais, comportamentais, físicas e sociais (Briere \& Elliot, 2003; Habigzang \& Caminha, 2004) que se prolongam na vida adulta (Fergusson, Bolden \& Horwood, 2008) e estão associadas à maior utilização de serviços de saúde (Chartier, Walker \& Naimark, 2010). Os efeitos deletérios do abuso sexual e os custos sociais e econômicos associados fazem com que venha sendo considerado um grave problema de saúde pública por ao menos três décadas (Browne \& Finkelhor, 1986; Kristensen, 1996; Finkelhor, Ormrod, Turner \& Hamby, 2005). Enquanto fenômeno crescente, apresenta-se como problemática de difícil enfrentamento por estar inserido num contexto histórico-cultural marcado por uma violência endêmica, de raízes muito profundas.

Do ponto de vista sociológico, o patriarcalismo e o sexismo são considerados os sistemas de poder que aprofundaram e legitimaram, ao longo da história, as violências entre gerações (Cantera, 2007), impondo às crianças e aos adolescentes uma condição, embora temporária, de submissão (Santos, 2005). As resultantes dessa visão verificam-se numa cultura do silêncio, na construção de mitos que naturalizaram tais práticas, criando empecilhos a uma ação pública para o seu enfrentamento (Fontes, 1993).

1 Endereço para correspondência: Av. Ipiranga, 6681 - Prédio 11, Sala 933 - Partenon - Porto Alegre/RS - CEP: 90619-900.
No Brasil, a Lei Federal 8.069/90 - o Estatuto da Criança e do Adolescente (ECA) adota a doutrina da proteção integral, concebendo a criança e o adolescente como sujeitos de direitos sociais, políticos e jurídicos (Brasil, 1990). O ECA prescreve, em seu artigo $5^{\circ}$, que "nenhuma criança ou adolescente será objeto de qualquer forma de negligência, discriminação, exploração, violência, crueldade e opressão, punido na forma da lei qualquer atentado, por ação ou omissão, aos seus direitos fundamentais" (Brasil, 1990).

Quando há suspeita de abuso sexual contra crianças e adolescentes, todos têm o dever de comunicar o fato às Autoridades policiais que, por sua vez, se encarregarão de investigar o caso. Ao mesmo tempo em que denúncias de abuso sexual contra crianças e adolescentes são cada vez mais frequentes, implicando em medidas protetivas urgentes para as vítimas e punições para os perpetradores, também são crescentes os casos de falsas denúncias. Portanto, profissionais de diferentes áreas têm atuado no estudo, na prevenção e na intervenção dos casos de abuso sexual, procurando compreender as implicações desse fenômeno e promovendo medidas de proteção para as vítimas (Caminha, 2000; Kristensen \& Schaefer, 2009). Pelo exposto, o presente artigo tem como principal objetivo revisar, de forma não sistemática, o papel da perícia psicológica no abuso sexual infantojuvenil com base na literatura nacional e internacional recente. 


\section{Violência contra crianças e adolescentes}

Nos últimos anos, observa-se um aumento nos estudos e na divulgação dos casos de violência contra crianças e adolescentes numa tentativa de desenvolver intervenções preventivas e de atenção ao problema (Polanczyck, Zavaschi, Benetti, Zenker \& Gammerman, 2003). Embora situações de violência sejam percebidas em diferentes culturas e classes sociais, os maus-tratos que atingem crianças e adolescentes podem passar silenciosamente despercebidos (Kristensen, Flores \& Gomes, 2003; Kristensen \& Schaefer, 2009; World Health Organization, 2006).

A questão da violência física e sexual envolvendo crianças e adolescentes ainda é fator de surpresa em nossa sociedade (Caminha, 2000). Soma-se a isso o despreparo, frequentemente observado, dos profissionais da área da saúde, educadores e juristas no manejo e tratamento adequados dos casos surgidos (Caminha, 2000). Os profissionais que atuam junto a crianças e adolescentes necessitam conhecer as características e os fatores de risco associados às situações abusivas, ressaltando-se a importância da compreensão da própria definição de abuso e maus-tratos e das consequências desses episódios no desenvolvimento infantojuvenil e psicopatológico (Polanczyck et al., 2003).

A Organização Mundial da Saúde (WHO, 2002) utiliza-se da definição de violência baseada na noção de que o ato violento se caracteriza pelo uso intencional de força ou poder, em situações de ameaça ou de uso efetivo, contra o próprio indivíduo ou outra pessoa, contra um grupo ou comunidade, que resulta ou possivelmente resultará em dano, morte, prejuízo psicológico, dificuldades de desenvolvimento ou privação. O termo maus-tratos contra crianças e adolescentes pode ser definido como ações intencionais ou por omissão ou qualquer atitude passiva ou negligente que lesione ou possa lesionar potencialmente a criança e/ou o adolescente, provocando danos que, de alguma forma, interfiram ou obstaculizem seu desenvolvimento físico, psicológico e/ ou social (Cesca, 2004; Leon, 2001; WHO, 1999). Quando tais ações são cometidas dentro ou fora de casa por algum membro da família, incluindo aqueles que não possuem laços de consanguinidade, mas exercem a função de cuidadores, são denominadas de violência intrafamiliar (Cesca, 2004; Habigzang \& Caminha, 2004; Kristensen, 2009).

Maus-tratos contra crianças e adolescentes são conceitualizados como uma forma de violência, repetitiva e intencional, na qual alguém (geralmente próximo à vítima) usa do poder e/ou da força física para envolvê-la em atos aos quais não está apta em nível biológico, psicológico ou cultural, resultando em danos reais ou potenciais para a saúde, desenvolvimento, sobrevivência ou dignidade da criança (Caminha, 2000; Flores, Kristensen \& Salzano, 1998; WHO, 1999). De acordo com Schreiber (2001), a definição de maus-tratos não é simples, pois muitas situações diferentes podem ser inseridas nesse conceito, incluindo a agressão física, a violência sexual, a negligência quanto à alimentação, saúde e proteção, a violência psicológica, o abandono físico e emocional, analisados sob o ponto de vista social, coletivo e institucional. A Síndrome de Münchausen por procuração e a exploração infantil também têm sido incluídas como formas de violência contra a criança ou adolescente (Habigzang
\& Caminha, 2004). Além disso, estudos apontam que, nas famílias abusadoras, é comum a coexistência dos diferentes tipos de violência (Kellog \& Menard, 2003; WHO, 2006).

\section{Abuso sexual}

O abuso sexual está entre uma das categorias mais comuns de maus-tratos contra crianças e adolescentes, as quais também incluem abuso físico, abuso emocional e negligência (Braun, 2002; Gonçalves, 2005; WHO, 1999; WHO, 2002). $\mathrm{O}$ abuso sexual pode ser definido como qualquer contato ou interação (como toques, carícias, sexo oral ou relações com penetração digital, genital ou anal, além de situações sem contato físico, como voyeurismo, assédio, exibicionismo, pornografia e exploração sexual) entre uma criança ou adolescente - que não estão aptos para compreender totalmente ou consentir com aquele ato - e alguém em estágio de desenvolvimento psicossexual mais avançado, na qual a criança ou adolescente estiver sendo usado para a estimulação sexual do perpetrador (Habigzang \& Caminha, 2004; Habigzang, Koller, Azevedo \& Machado, 2005; Kristensen, 1996; WHO, 1999, 2002).

As consequências decorrentes do abuso sexual variam desde efeitos mínimos até problemas mais graves, com repercussões sociais, emocionais e/ou psiquiátricas - como depressão, transtornos de ansiedade (entre os quais, o Transtorno de Estresse Pós-Traumático), transtornos alimentares, transtornos dissociativos, Transtorno de Déficit de Atenção/ Hiperatividade e, até mesmo, Transtorno da Personalidade Borderline (Briere \& Elliot, 2003; Cicchetti \& Toth, 2005; Cohen \& Mannarino, 2000; Collin-Vézina \& Hébert, 2005; Nurcombe, 2000; Saywitz, Mannarino, Berliner \& Cohen, 2000). Também é descrita a manifestação de sintomas como: tristeza, ideação suicida, medo exagerado de adultos, comportamento sexual avançado para a idade, masturbação frequente e/ou pública, baixa autoestima, abuso de substâncias químicas, sonolência, enurese, encoprese, tiques e manias, isolamento social, dificuldades de aprendizagem, irritabilidade, entre outros (Gerko, Hughes, Hamil \& Waller, 2005; Meyerson, Long, Miranda Jr. \& Marx, 2002; Pfeiffer \& Salvagni, 2005; Polanczyck et al., 2003; Ystgaard, Hestetun, Loeb \& Mehlum, 2004).

Fatores intrínsecos à criança (funcionamento psíquico, idade, sentimentos de culpa/responsabilização) bem como fatores extrínsecos a ela, como situações de risco, diferença de idade e grau de relação entre perpetrador e vítima, tipo de atividade sexual, violência e ameaças sofridas, número de situações abusivas vivenciadas (duração do abuso), reação dos outros, suporte familiar, econômico e social (incluindo acesso a tratamento adequado e especializado) têm sido relacionados a essa variedade de respostas (Ehlers, Mayou \& Bryant, 2003; Furniss, 1993; Kristensen, 1996; Saywitz el at., 2000; Shore, 2002; WHO, 2002).

\section{Abuso sexual intrafamiliar}

Pesquisas epidemiológicas realizadas apontaram que $80 \%$ dos casos de abuso sexual contra crianças e adolescentes eram 
perpetrados no contexto doméstico, tendo duração mínima de um ano (Braun, 2002; Kristensen, Oliveira \& Flores, 1999). Nessas situações em que os episódios são praticados por pessoas próximas às vítimas, os abusos são denominados de intrafamiliares ou incestuosos (Braun, 2002; Furniss, 1993; Habigzang \& Caminha, 2004).

Numa tentativa de compreender o fenômeno da violência intrafamiliar, autores observaram, entre os membros dessas famílias, relações hierarquicamente assimétricas caracterizadas por desigualdade e subordinação (Furniss, 1993; Koller, 1999; WHO, 2006). Além disso, verificaram que muitos indivíduos que atuavam como perpetradores da violência já haviam sido vítimas de alguma forma de maus-tratos durante sua infância e/ou adolescência (Kristensen, 1996). Nos casos de violência intrafamiliar, são comumente observados desajustes familiares e problemas psíquicos, alcoolismo e aspectos sociais, econômicos e culturais (Banyard, Williams \& Siegel, 2004; Habigzang \& Caminha, 2004). Entretanto, deve-se atentar para não generalizar tais achados, entendendo-os como fatores que podem contribuir para o desencadeamento ou a manutenção de situações de abuso, não devendo, portanto, serem percebidos numa relação direta de causa e efeito (Caminha, 2000; Habigzang \& Caminha, 2004).

$\mathrm{Na}$ dinâmica do abuso sexual infantojuvenil, é comum que ocorra a retratação, a negação ou a dissociação, contribuindo para a perpetuação da violência por um longo período e impedindo a sua revelação, ou seja, fortalecendo a Síndrome de Segredo no sistema familiar (Azambuja, 2004; Dobke, 2001). Muitas vezes a vítima tenta revelar a situação abusiva, sendo a revelação interpretada como imaginação ou mentira da criança (Kristensen et al., 2003).

\section{Perícia psicológica: algumas considerações}

A palavra perícia provém do latim peritia e significa habilidade, destreza, vistoria ou exame de caráter técnico e especializado (Rodrigues, 2004; Rovinski, 2007). O principal objetivo da perícia é fazer prova, ou seja, investigar e definir como ocorreu determinado fato, confirmando a sua exatidão (Dorea, Stumvoll \& Quintela, 2005).

No ordenamento jurídico brasileiro, embora não haja hierarquização entre os diferentes tipos de provas (material, documental ou testemunhal), a prova pericial/material, por estar fundamentada em bases científicas, tem destaque em relação às demais. No artigo 158 do Código de Processo Penal (CPP), é dito que "quando a infração deixar vestígios, será indispensável o exame de corpo de delito, direto ou indireto, não podendo supri-lo a confissão do acusado", sendo que, no artigo seguinte, é referido que "o exame de corpo de delito e outras perícias serão realizados por perito oficial, portador de diploma de curso superior" (Brasil, 1941; 2008).

Os peritos são profissionais nomeados pelo juiz ou oficialmente constituídos por concurso público para realizarem a perícia, sendo pessoas com entendimento técnico do assunto que fornecem a sua verificação e interpretação dos fatos, emitidos através do laudo (Benfica \& Vaz, 2008). Os profissionais designados para as atividades periciais necessitam conhecimentos técnico-científicos especializados, que os possibilitem compreender e distinguir os fatos investigados (Dorea et al., 2005).

A perícia psicológica é aquela realizada por psicólogos com o intuito de responder a um questionamento jurídico (Arantes, 2005; Cesca, 2004; Rovinski, 2007). Além de possuir conhecimentos técnicos e teóricos específicos sobre a sua área de atuação, o psicólogo deve estar a par da terminologia e da legislação vigente, estabelecendo objetivos de avaliação e construindo procedimentos que sejam legalmente relevantes, orientando seu trabalho aos propósitos judiciais (Brandão, 2005; Rodrigues, 2004; Rovinski, 2007). Para isso, é importante uma integração entre os conhecimentos da área de saúde mental e do Direito, visto que embora haja muitas diferenças entre esses saberes, ambos têm como objetivo comum fazer com que o indivíduo e a sociedade possam ter um melhor convívio (Cohen, 2006).

O cliente do psicólogo será tanto o sujeito periciado como o sistema mais amplo, ou seja, a sociedade (Echeburúa \& Subijana, 2008; Rovinski, 2007). Em virtude disso, é necessário que o perito esclareça seu papel e solicite o consentimento do examinado, ressaltando que as informações prestadas serão encaminhadas à autoridade requerente (Echeburúa \& Subijana, 2008). Portanto, os resultados da avaliação nem sempre estarão de acordo com os interesses do periciado (Heilbrun et al., 2003).

A perícia psicológica abrange a entrevista, a seleção, a aplicação e o levantamento de testes e de fatos da vida referentes ao passado e ao presente do sujeito e do episódio ocorrido, de acordo com as necessidades e questões levantadas em cada processo. Exige do psicólogo, portanto, a capacidade de integrar as informações obtidas a partir de diferentes fontes em um relatório coerente e consistente. Convém ressaltar que os instrumentos empregados pelo psicólogo devem obedecer à determinação do órgão máximo profissional, o Conselho Federal de Psicologia (Rodrigues, 2004).

A avaliação forense distingue-se de outras modalidades de avaliação, como por exemplo, nas situações com fins diagnósticos ou de planejamento do tratamento (Heilbrun et al., 2003). No trabalho pericial, o avaliador assume um papel objetivo ou quase objetivo, devendo fornecer informações com base empírica, exigindo-se um padrão elevado de precisão e relevância dos dados coletados (Heilbrun et al., 2003).

Heilbrun (2001 conforme citado em Heilbrun et al., 2003) organizou princípios que orientam a avaliação forense de saúde mental, distribuídos nas seguintes etapas: preparação, coleta de dados, interpretação dos dados e comunicação. Entre esses princípios, o autor preconiza que o profissional não tome parte em perícias nas quais sua imparcialidade seja improvável, não se permita desempenhar o papel de terapeuta juntamente com o de perito, leve em consideração múltiplas fontes de dados, obtenha informações históricas pertinentes relativas ao periciado, garanta a ausência de distrações e demais influências no ambiente de avaliação e registre seus achados de forma detalhada e completa.

O laudo psicológico deve ser claro, conciso e harmônico (Conselho Federal de Psicologia, 2003). Esse documento estruturado tem por finalidade relatar de maneira descritiva, metódica, fiel e objetiva os dados coletados durante a perícia (Heilbrun et al., 2003). Nele, o psicólogo emitirá conclusões acerca do estudo realizado e dos quesitos e questionamentos 
previamente formulados, devendo responder a todas as informações que lhe foram solicitadas com o emprego de uma linguagem simples, evitando o jargão técnico (Heilbrun et al., 2003; Rodrigues, 2004).

\section{Perícia psicológica em casos de abuso sexual}

Na identificação do abuso sexual, um grande problema é que atos libidinosos podem não deixar vestígios, assim como marcas decorrentes de abuso físico podem desaparecer até a data em que for realizada a perícia médica (Dobke, 2001; Echeburúa \& Subijana, 2008; Magalhães et al., 1998). Na ausência de provas físicas, não há um indicador específico que determine se uma criança foi sexualmente abusada (Herman, 2005) e, em virtude disso, especialistas ressaltam que análises abrangentes são as mais indicadas, já que uma boa avaliação também reflete um processo integrado que vai muito além de qualquer pontuação num teste isolado (Finnilä-Tuohimaa, Santtila, Sainio, Niemi \& Sandnabba, 2009; Friedrich, 2001).

É consenso, entre os profissionais da área da saúde, que a identificação de situações de crianças e adolescentes vítimas de abuso sexual é um desafio. Um dos motivos alegados é que a maioria dos casos envolve crianças muito pequenas, com limitações na comunicação verbal, compreensão limitada e a presença de ansiedade excessiva (Friedrich, 2001). Além disso, sobretudo nos casos de abuso intrafamiliar, a criança frequentemente não consegue diferenciar aquele ato praticado pelo cuidador, que deveria ser responsável pela sua proteção e pela promoção de suas necessidades básicas, como uma forma de abuso.

A perícia psicológica realizada em casos de abuso sexual de crianças e adolescentes deve incluir entrevistas com os responsáveis e com a vítima (Friedrich, 2001). Uma possibilidade é realizar alguma dessas entrevistas conjuntamente, com vistas à observação da dinâmica familiar (Rovinski, 2007). Nesses casos, o psicólogo adquire um papel de julgador, devendo atentar para o grau de incongruência entre as necessidades da criança e as habilidades parentais despendidas (Rovinski, 2007). O psicólogo destaca e analisa os aspectos psicológicos das pessoas envolvidas, ocultos por trás das relações processuais. O principal objetivo da perícia psicológica é auxiliar o juiz na tomada de uma decisão, garantindo, consequentemente, os direitos e o bem-estar da criança e/ou adolescente (Silva, 2003).

É necessário que se atente para o maior número possível de elementos disponíveis, como a coleta do relato da situação vivenciada, a análise das repercussões físicas e psicológicas, entrevistas com os responsáveis, registros escolares, entre outros, a fim de que se obtenham conclusões confiáveis com relação às situações relatadas (Welter \& Feix, 2010). Também é importante considerar se a situação descrita é condizente com experiências narradas por outras vítimas, se o afeto é correspondente ao conteúdo da verbalização, se o estilo de exposição se modifica quando o assunto específico da situação abusiva é introduzido e se há evidências de treinamento, indução, sugestão ou alguma motivação para aquela denúncia (Heiman, 1992).
A entrevista psicológica é uma técnica privilegiada no processo de perícia, pois possibilita, através da análise da comunicação verbal e não verbal, conhecer dados provenientes de diversas fontes. Portanto, deve ser realizada em um ambiente protegido e empático, permitindo a manifestação das emoções e dos pensamentos do periciado, isentando-o da culpa (Echeburúa \& Subijana, 2008). É preciso que o perito considere a faixa etária da vítima, bem como a situação emocional desencadeada pelo possível abuso vivenciado (Echeburúa \& Subijana, 2008). Conforme o protocolo empregado pela Universidade de Michigan (Faller, 2003), a estrutura da entrevista deve contemplar, basicamente, três etapas: a etapa inicial (rapport, estabelecimento das regras básicas da entrevista, avaliação do nível de desenvolvimento do avaliado, bem como se o menor sob avaliação consegue distinguir realidade e fantasia, investigação de outras questões sobre a sua vida), a etapa focalizada no abuso (empregar perguntas abertas, indagar se o examinado conhece o motivo pelo qual está ali, estimular o relato livre e desenvolver questionamentos a partir do que é emitido) e a etapa do encerramento (informar ao periciado sobre os próximos passos a serem tomados, colocar-se disponível, ajudá-lo a restabelecer o equilíbrio, incluindo a manifestação de sentimentos, pensamentos e atitudes em relação à revelação e a situação vivenciada).

Dessa maneira, o relato da vítima constitui, na maioria das vezes, um dos principais elementos de prova (Daltoé Cezar, 2007; Herman, 2010; Magalhães \& Ribeiro, 2007; Stein, Pergher \& Feix, 2009; Werner \& Werner, 2008) e, por sua importância no processo, há grande ênfase, atualmente, na credibilidade do testemunho da vítima (Welter \& Feix, 2010). Logo, a análise dos critérios de confiabilidade e validade do relato emitido pela criança ou adolescente a respeito da suposta situação abusiva vivenciada mostra-se fundamental (Echeburúa \& Subijana, 2008; Rovinski, 2007). Um método que tem sido amplamente utilizado é o Statement Validity Assessment (SVA; Steller \& Boychuk, 1992), que é composto por três etapas: entrevista semiestruturada, análise de conteúdo baseada em critérios (Criteria-Based Content Analysis - CBCA), em que se avalia a presença de critérios específicos considerados indicadores de credibilidade, e a lista de controle de validade dos resultados obtidos pelo CBCA (Vrij, 2005).

Apesar da avaliação pericial não ter como objetivo atuar como uma intervenção psicossocial e estabelecer vínculo terapêutico, a possibilidade de relatar a situação abusiva num ambiente neutro, seguro, confiável e com técnicos capacitados para evitar possíveis sugestões e abusos, pode constituir uma experiência extremamente benéfica para a vítima, que se torna parte ativa do processo e consciente de seus direitos (Silva Júnior, 2006). A emissão do relato pode ativar e reorganizar a memória traumática, além de reestruturar crenças distorcidas sobre culpa, autoimagem e diferenças em relação aos pares (Friedrich, 2001; Habigzang, Corte, Hatzenberger, Stroeher \& Koller, 2008). A partir de uma pesquisa realizada com meninos vítimas de abuso sexual, Kristensen et al. (2003) relatam que a valorização do processo comunicativo utilizado nas entrevistas iniciais acaba desempenhando, também, uma função terapêutica, na medida em que traz para o plano reflexivo a percepção das 
reações experienciadas, bem como os contextos, a revelação e os prejuízos da situação abusiva, entre outros aspectos. Além disso, nesse estudo, foi constatado que o processo de revelação estava diretamente relacionado com a noção das vítimas sobre as consequências futuras desse ato.

Além de entrevistas com as vítimas e responsáveis, o uso complementar de testes psicológicos é um procedimento bastante utilizado, cujo objetivo é buscar sinais e sintomas cognitivos, emocionais e comportamentais compatíveis com a ocorrência do abuso sexual (Fortes, Scheffer \& Kapczinsky, 2007). No Brasil, os psicólogos devem selecionar, a partir dos instrumentos de psicologia reconhecidos pelo Conselho Federal de Psicologia, aqueles que mais atendam à criança e/ ou ao adolescente, levando em consideração alguns critérios como a idade, as características da vítima e as circunstâncias de cada caso (Magalhães \& Ribeiro, 2007).

Convém ressaltar que não existem instrumentos psicológicos específicos direcionados para a constatação da violência sexual (Herman, 2005). Portanto, destaca-se prudência na busca por manifestações comportamentais compatíveis com essa vitimização, tendo em vista que muitos sintomas são inespecíficos e podem estar presentes em outros quadros, produzindo falso-positivos (Friedrich, 2001). Em virtude disso, é relevante uma análise da causalidade (nexo causal) entre o evento traumático experienciado e os sintomas manifestados, avaliando a presença de outros aspectos no histórico e no contexto de vida da criança que podem estar contribuindo para o quadro sintomatológico. Recursos lúdicos também são utilizados (Faller, 2003), visto que a brincadeira pode auxiliar o vínculo e fornecer indicadores sobre quais aspectos necessitam de investigações adicionais, além de corroborar impressões prévias sobre o nível de desenvolvimento e o funcionamento geral da criança.

De maneira geral, destaca-se a importância dos peritos respeitarem o ritmo de discurso da vítima e não emitirem julgamentos sobre o que está sendo relatado (Magalhães \& Ribeiro, 2007). Devem estar atentos para a comunicação não verbal, para a tonalidade emocional e para a postura, aceitando possíveis incoerências, lacunas de informação e contradições no discurso do periciado (Magalhães \& Ribeiro, 2007). Além disso, deve-se cuidar para não contaminar os dados fornecidos pela vítima, criando falsas memórias (Belli \& Loftus, 1995).

Estudos têm investigado o processo de falsas memórias no relato de testemunhos, identificando que perguntas feitas de forma tendenciosa podem influenciar o relato, conduzindo a falsas confissões e a informações equivocadas, resultando, consequentemente, em depoimentos inverídicos (Ceci \& Huffman, 1997; Garven, Wood, Malpass \& Shaw, 1998; Stein et al., 2009). Deve-se, portanto, fazer perguntas abertas, não tendenciosas, de forma a não influenciar a vítima (Felix \& Pergher, 2010; Stein et al., 2009).

Ao mesmo tempo, na realização da perícia psicológica, é preciso estar atento para a possibilidade de falsas denúncias, como em casos de divórcio, disputas de guarda, pensão alimentícia e alienação parental, situações nas quais um dos cuidadores pode influenciar a criança ou o adolescente a relatar uma situação abusiva com o intuito de prejudicar o genitor que está sendo acusado ou para obter algum tipo de vantagem (Echeburúa \& Subijana, 2008; Faller, 2003; Gard- ner, 2002; Guazzelli, 2007; Trocmé \& Bala, 2005; Simão, 2007). Assim, o psicólogo necessita constantemente atentar para esses inúmeros aspectos, considerando, também, que manipular a criança ou o adolescente a dar um depoimento falso pode ser considerado um tipo de violência e, inclusive, acarretar em sérias implicações para o seu desenvolvimento (Guazzelli, 2007). Portanto, a atividade pericial requer tanto conhecimento aprofundado no assunto, quanto boa capacidade de tomada de decisão (Finnilä-Tuohimaa et al., 2009).

Atualmente, tem se discutido sobre a escuta de crianças e adolescentes vítimas de situações abusivas (Conselho Federal de Psicologia, 2010). Com a intenção de minimizar os impactos do depoimento da vítima em juízo, foram propostas alternativas para que a referida escuta seja realizada em ambiente mais adequado, humanizado e acolhedor (Daltoé Cezar, 2007). Especificamente na avaliação pericial, conforme já mencionado, o psicólogo considera, além da escuta das vítimas e dos responsáveis, uma análise da sintomatologia e do contexto, identificando possíveis pressões e/ou motivações para depoimentos inverídicos e, até mesmo, a possibilidade de produzir falsas memórias. É essencial que o profissional possa obter o discurso do periciado de forma neutra, deparando-se com um relato livre, minimizando, dessa maneira, a possibilidade de contaminação ou sugestionabilidade. Também é relevante entender o momento e o contexto em que ocorreram a denúncia e a revelação, compreendendo se esta última decorreu do relato espontâneo da criança ou se foi verbalizada a partir de questionamentos de adultos (familiares, outros profissionais, etc.).

Percebe-se, portanto, que o depoimento da criança em juízo (prova testemunhal) e a perícia psicológica (prova material/pericial) não são procedimentos equivalentes, visto que possuem peculiaridades próprias e ocorrem em momentos distintos. Apesar disso, ambas as ferramentas são importantes, complementares e têm como objetivo comum auxiliar no esclarecimento do caso.

\section{Considerações Finais}

A perícia psicológica, considerada um meio de prova, é uma modalidade de avaliação técnica e especializada comumente empregada na investigação de situações abusivas contra crianças e adolescentes, tendo em vista que, em muitos casos, não há materialidade suficiente que possa ser constatada por meio de um exame médico-legal (Dobke, 2001; Echeburúa \& Subijana, 2008; Magalhães et al., 1998; Silva Júnior, 2006). A escuta das crianças nesses contextos é essencial e, em muitos casos, decisiva na constituição da prova judicial que conduzirá o processo (Herman, 2010; Daltoé Cezar, 2007).

Apesar de não existirem instrumentos específicos e indicadores precisos para a constatação do abuso sexual, a perícia psicológica é uma das maneiras de acessar o histórico do examinando e sua sintomatologia, descartando outras ocorrências que possam ter desencadeado o quadro sintomático avaliado. Uma avaliação abrangente e compreensiva demonstra-se, portanto, imprescindível, devendo basear-se na integração de diferentes fontes de informação e de muitos indicadores (Friedrich, 2001; Hershkowitz \& Terner, 2007). 
Mesmo que não se configure como uma intervenção psicossocial, o processo de avaliação deve ser desenvolvido com um nível de atenção ao bem-estar do cliente suficiente para que a avaliação não seja prejudicial ao periciado ou ao processo de investigação. Ainda que isenta de objetivos terapêuticos, a avaliação pericial pode produzir alterações positivas na recuperação da vítima, na medida em que se constitui num momento de escuta e de proteção (Magalhães \& Ribeiro, 2007). Consequentemente, um dos maiores cuidados do examinador deve centrar-se em não tornar a entrevista um elemento abusivo, preocupando-se em não revitimizar a criança ou o adolescente, tendo em vista que a denúncia e a consequente ação judicial podem agravar o sofrimento psicológico já experienciado (Daltoé Cezar, 2007; Dobke, 2001; Echeburúa \& Subijana, 2008).

Alguns fatores como o sexo do examinador, a idade da vítima ou características específicas da maneira como a entrevista é conduzida parecem influenciar o processo de revelação dos episódios de abuso sexual durante as avaliações forenses (Lippert, Cross, Jones \& Walsh, 2009). Entre os principais desafios na perícia psicológica do abuso sexual, estão os casos que envolvem crianças em idade pré-escolar, cuja forma de comunicação é predominantemente não verbal. Além disso, quais os recursos disponíveis para avaliar crianças ou adolescentes que não relatam a situação abusiva para o perito? Sabe-se que a dinâmica do abuso sexual inclui fenômenos como a Síndrome do Segredo, a negação e a retratação. Seria possível, então, concluir, a partir do silêncio da criança, que ela não está sofrendo maus-tratos?

Esforços devem ser realizados a fim de aprimorar e desenvolver técnicas de entrevistas, instrumentos e procedimentos de avaliação válidos para este contexto e para esta população. É primordial o incentivo à formação técnico-científica dos profissionais de psicologia que atuam na área forense, através de programas de educação continuada e projetos associados a Instituições de Ensino e Pesquisa.

Atuar em casos de violência sexual contra crianças e adolescentes é um desafio que requer conhecimento técnico, preparo emocional, sensibilidade, atenção e articulação entre os diferentes sistemas envolvidos (Fröner \& Ramires, 2008; 2009; Habigzang, Ramos \& Koller, 2011). A partir disso, será possível assegurar, de maneira efetiva e interdisciplinar, a proteção integral da criança, garantindo os seus direitos e respeitando a sua condição peculiar de sujeito em desenvolvimento, conforme promulgado pelo ECA (Brasil, 1990). Conhecer a realidade sobre a violência sexual e suas implicações no desenvolvimento de crianças e adolescentes é fator indispensável para a sensibilização e a compreensão desse fenômeno, bem como para a elaboração de estratégias de intervenção ou ação necessárias que visem romper a indiferença frente essa realidade (Laks, Werner \& Miranda-Sá Jr., 2006).

\section{Referências}

Arantes, E. M. M. (2005). Pensando a Psicologia aplicada à Justiça. In H. S. Gonçalves, \& E. P. Brandão (Eds.), Psicologia Jurídica no Brasil (pp. 15-49). Rio de Janeiro: Nau.
Azambuja, M. R. F. (2004). Violência Sexual Intrafamiliar: é possivel proteger a criança? Porto Alegre: Livraria do Advogado.

Banyard, V. L., Williams, L. M., \& Siegel, J. A. (2004). Childhood sexual abuse: a gender perspective on context and consequences. Child Maltreatment, 9(3), 223-238.

Belli, R. F., \& Loftus, E. F. (1995). The pliability of autobiographical memory: misinformation and the false memory problem. In D. C. Rubin (Ed.), Remembering our past: studies in autobiographical memory. Cambridge: Cambridge University Press.

Benfica, F. S., \& Vaz, M. (2008). Medicina Legal. Porto Alegre: Livraria do Advogado.

Brandão, E. P. (2005). A interlocução com o Direito à luz das práticas psicológicas em Varas de Família. In H. S. Gonçalves \& E. P. Brandão (Eds.), Psicologia Jurídica no Brasil (pp. 51-97). Rio de Janeiro: Nau.

Brasil (1941). Decreto-lei no 3.689, de 3 de outubro de 1941. Código de Processo Penal. Diário Oficial da União, Brasília (DF).

Brasil (1990). Lei no 8.069, de 13 de julho de 1990. Estatuto da Criança e do Adolescente. Diário Oficial da União, Brasília (DF).

Brasil (2008). Lei $n^{0} 11.690$, de 9 de junho de 2008. Altera dispositivos do Decreto-Lei no 3.689 , de 3 de outubro de 1941 - Código de Processo Penal, relativos à prova, e dá outras providências. Diário Oficial da União, Brasília (DF).

Braun, S. A. (2002). A violência sexual infantil na familia: do silêncio à revelação. Porto Alegre: Age.

Briere, J., \& Elliot, D. M. (2003). Prevalence and psychological sequel of self-reported childhood physical and sexual abuse in a general population sample of men and women. Child Abuse \& Neglect, 27(10), 1205-1222.

Browne, A., \& Finkelhor, D. (1986). Impact of child sexual abuse: a review of the research. Psychological Bulletin, 99, 66-77.

Caminha, R. M. (2000). A violência e seus danos à criança e ao adolescente. In AMENCAR (Ed.), Violencia Doméstica (pp. 43-60). Brasília: UNICEF.

Cantera, L. (2007). Casais e violência: um enfoque além do gênero. Porto Alegre: Dom Quixote.

Ceci, S., \& Huffman, M. L. C. (1997). How suggestible are preschool children? Cognitive and social factors. Journal by the American Academy of Child and Adolescent Psychiatry, 36(7), 948-958.

Cesca, T. B. (2004). O papel do psicólogo jurídico na violência intrafamiliar: possíveis articulações. Psicologia \& Sociedade, 16(3), 41-46.

Chartier, M. J., Walker, J. R., \& Naimark, B. (2010). Separate and cumulative effects of adverse childhood experiences in predicting adult health and health care utilization. Child Abuse \& Neglect, 34, 454-464.

Cicchetti, D., \& Toth, S. L. (2005). Child maltreatment. Annual Review of Clinical Psychology, 1, 409-438.

Cohen, C. (2006). O profissional de saúde mental no tribunal. In C. Cohen, M. Segre, \& F. C. Ferraz (Eds.), Saúde mental, crime e justiça (2a. ed.) (pp. 239-246). São Paulo: Edusp.

Cohen, J. A., \& Mannarino, A. P. (2000). Predictors of treatment outcome in sexually abused children. Child Abuse \& Neglect, 24(7), 983-994. 
Collin-Vézina, D., \& Hébert, M. (2005). Comparing dissociation and PTSD in sexually abused school-aged girls. Journal of Nervous and Mental Disease, 193(1), 47-52.

Conselho Federal de Psicologia (2003). Resolução CFP 007/2003. Conselho Federal de Psicologia (2010). Resolução CFP 010/2010.

Daltoé Cezar, J. A. (2007). A inquirição de crianças vítimas de abuso sexual em juízo. In M. B. Dias (Ed.), Incesto e alienação parental: realidades que a Justiça insiste em não ver (pp. 169186). São Paulo: Editora Revista dos Tribunais.

Dobke, V. (2001). Abuso sexual: A inquirição das crianças, uma abordagem interdisciplinar. Porto Alegre: Ricardo Lenz.

Dorea, L. E. C., Stumvoll V. P. E., \& Quintela, V. (2005). Criminalística (3a. ed.). Campinas: Millenium.

Echeburúa, E., \& Subijana, I. J. (2008). Guía de buena práctica psicológica en el tratamiento judicial de los niños abusados sexualmente. Int J Clin Health Psychol, 8(3), 733-749.

Ehlers, A., Mayou, R. A., \& Bryant, B. (2003). Cognitive predictors of posttraumatic stress disorder. Behaviour Research and Therapy, 38(4), 319-345.

Faller, K. C. (2003). Understanding and assessing child sexual maltreatment (2a. ed.). Thousand Oaks, CA: Sage.

Felix, L. da F., \& Pergher, G. K. (2010). Memória em julgamento: Técnicas de entrevista para minimizar as falsas memórias. In L. M. Stein (Ed.), Falsas memórias: Fundamentos científicos e suas aplicações clínicas e jurídicas (pp. 209-227). Porto Alegre: Artmed.

Fergusson, D. M., Bolden, J. M., \& Horwood, L. J. (2008). Exposure to childhood sexual and physical abuse and adjustment in early adulthood. Child Abuse \& Neglect, 32, 607-619.

Finkelhor, D., Ormrod, R., Turner, H., \& Hamby, S. L. (2005). The victimization of children and youth: a comprehensive, national survey. Child Maltreatment, 10, 5-25.

Finnilä-Tuohimaa, K., Santtila, P., Sainio, M., Niemi, P., \& Sandnabba, K. (2009). Expert judgment in cases of alleged child sexual abuse: clinicians' sensitivity to suggestive influences, pre-existing beliefs and base rate estimates. Scandinavian Journal of Psychology, 50, 129-142.

Flores, R. Z., Kristensen, C. H., \& Salzano, F. M. (1998). Definir e medir o que são abusos sexuais. In M. de F. Pinto Leal \& M. A. César (Eds.), Indicadores de violência intra-familiar e exploração sexual comercial de crianças e adolescentes (pp. 39-55). Brasília: Cese/Ministério da Justiça.

Fontes, L. A. (1993). Considering culture and oppression: steps toward an ecology of sexual child abuse. Journal of Feminist Family Therapy, 5, 25-54.

Fortes, M. G. C., Scheffer, M. S., \& Kapczinsky, N. S. (2007). Elementos indicativos de abuso sexual na infância obtidos pelo método Rorschach. Revista HCPA, 27(3), 5-12.

Friedrich, W. N. (2001). Psychological assessment of sexually abused children and their families. Thousand Oaks, CA: Sage.

Fröner, J. P., \& Ramires, V. R. R. (2008). Escuta de crianças vítimas de abuso sexual no âmbito jurídico: Uma revisão crítica da literatura. Padéia, 18(40), 267-278.

Fröner, J. P., \& Ramires, V. R. R. (2009). A escuta de crianças vítimas de abuso sexual intrafamiliar na concepção de profissionais que atuam no âmbito do Judiciário. Psicologia em Revista, 15(3), 60-81.

Furniss, T. (1993). Abuso sexual da criança: Uma abordagem multidisciplinar. Porto Alegre: Artes Médicas.
Gardner, R. (2002). Parental alienation syndrome vs. parental alienation: Which diagnosis should evaluators use in childcustody disputes? American Journal of Family Therapy, 30, 93-115.

Garven, S., Wood, J. M., Malpass, R. S., \& Shaw, J. S. (1998). More than suggestion: The effect of interviewing techniques from the McMartin preschool case. Journal of Applied Psychology, 83, 347-359.

Gerko, K., Hughes, M. L., Hamil, M., \& Waller, G. (2005). Reported childhood sexual abuse and eating-disordered cognitions and behavior. Child Abuse \& Neglect, 29 (4), 375-382.

Gonçalves, H. S. (2005). Violência contra a criança e o adolescente. In H. S. Gonçalves \& E. P. Brandão (Eds.), Psicologia Jurídica no Brasil (pp. 277-307). Rio de Janeiro: Nau.

Guazzelli, M. (2007). A falsa denúncia de abuso sexual. In M. B. Dias (Ed.), Incesto e alienação parental: Realidades que a Justiça insiste em não ver (pp. 112-139). São Paulo: Editora Revista dos Tribunais.

Habigzang, L. F., \& Caminha, R. M. (2004). Abuso sexual contra crianças e adolescentes: conceituação e intervenção clínica. São Paulo: Casa do Psicólogo.

Habigzang, L. F., Corte, F. D., Hatzenberguer, R., Stroeher, F., \& Koller, S. H. (2008). Avaliação psicológica em casos de abuso sexual na infância e adolescência. Psicologia: Reflexão e Crítica, 21(2), 338-344.

Habigzang, L. F., Koller, S., Azevedo, G., \& Machado, P. (2005). Abuso sexual infantil e dinâmica familiar: Aspectos observados em processos jurídicos. Psicologia: Teoria e Pesquisa, 21(3), 341-348.

Habigzang, L. F., Ramos, M. S., \& Koller, S. (2011). A revelação de abuso sexual: As medidas adotadas pela rede de apoio. Psicologia: Teoria e Pesquisa, 27(4), 467-473.

Heilbrun, K., Marczyk, G. R., DeMatteo, D., Zillmer, E. A., Harris, J., \& Jennings, T. (2003). Principles of forensic mental health assessment: Implications for neuropsychological assessment in forensic contexts. Assessment, 10(4), 329-343.

Heiman, M. L. (1992). Putting the puzzle together: Validating allegations of child sexual abuse. Journal of Child Psychology and Psychiatry, 33, 311-329.

Herman, S. (2005). Improving decision making in forensic child sexual abuse evaluations. Law and Human Behavior, 29(1), 87-120.

Herman, S. (2010). The role of corroborative evidence in child sexual abuse evaluations. Journal of Investigative Psychology and Offender Profiling, 7, 189-212.

Hershkowitz, I., \& Terner, A. (2007). The effects of repeated interviewing on children's forensic statements of sexual abuse. Applied Cognitive Psychology, 21, 1131-1143.

Kellog, N. D., \& Menard, S. W. (2003). Violence among family members of children and adolescents evaluated for sexual abuse. Child Abuse \& Neglect, 27, 1367-1376.

Koller, S. H. (1999). Violência doméstica: uma visão ecológica. In AMENCAR (Ed.), Violência doméstica (pp. 32-42). Brasília: UNICEF.

Kristensen, C. H. (1996). Abuso sexual em meninos. Dissertação de Mestrado, Universidade Federal do Rio Grande do Sul, Porto Alegre. 
Kristensen, C. H. (2009). Transtorno de Estresse Pós-Traumático. In V. G. Haase, F. O. Ferreira, \& F. J. Penna (Eds.), Aspectos biopsicossociais da saúde na infância e adolescência (pp. 259-272). Belo Horizonte: Coopmed.

Kristensen, C. H., Flores, R. Z., \& Gomes, W. B. (2003). Revelar ou não revelar: Uma abordagem fenomenológica do abuso sexual com crianças. In M. A. T. Bruns \& A. F. Holanda (Eds.), Psicologia e pesquisa fenomenológica: reflexões e perspectivas (pp. 111-142). São Paulo: Ômega.

Kristensen, C. H., Oliveira, M. S., \& Flores, R. Z. (1999). Violência contra crianças e adolescentes na Grande Porto Alegre: pode piorar? In AMENCAR (Ed), Violência doméstica (pp. 104117). São Leopoldo.

Kristensen, C. H., \& Schaefer, L. S. (2009). Maus-tratos na infância e adolescência. In I. M. C. C. de SOUZA(Ed.), Parentalidade: Análise psicojurídica (pp. 183-208). Curitiba: Juruá.

Laks, J., Werner, J., \& Miranda-Sá Jr. L. S. de. (2006). Psiquiatria forense e Direitos Humanos nos pólos da vida: Crianças, adolescentes e idosos. Revista Brasileira de Psiquiatria, 28 (supl. II), s80-5.

Leon, I. A. (2001). Violencia y sexualidad. Habana: Editorial Nuevo Milênio.

Lippert, T., Cross, T. P., Jones, L., \& Walsh, W. (2009). Telling interviewers about sexual abuse: Predictors of child disclosure at forensic interviews. Child Maltreatment, 14(1), 100-113.

Magalhães, T., \& Ribeiro, C. (2007). A colheita de informações a vítimas de crimes sexuais. Acta Med Port, 20, 439-445.

Magalhães, T., Souza, M. J. C. de, Silva, A. G. da, Costa, D. P. da., Grams, A. C., Ribeiro, C., Gonzalez, R., \& Costa, J. P. da. (1998). Child sexual abuse: a preliminary study. Journal of Clinical Medicine, 111(5), 1-7.

Meyerson, L. A., Long, P., Miranda Jr., R., \& Marx, B. P. (2002). The influence of childhood sexual abuse, physical abuse, family environment, and gender on the psychological adjustment of adolescents. Child Abuse \& Neglect, 26, 387-405.

Nurcombe, B. (2000). Child sexual abuse I: Psychopathology. Australian and New Zealand Journal of Psychiatry, 34(1), 85-91.

Pfeiffer, L., \& Salvagni, E. P. (2005). Visão atual do abuso sexual na infância e adolescência. Jornal de Pediatria, 81(5), 197-204.

Polanczyck, G., Zavaschi, M. L., Benetti, S. P. C., Zenker, R., \& Gammerman, P. (2003). Violência sexual e sua prevalência em adolescentes de Porto Alegre, Brasil. Revista de Saúde Pública, 37(1), 8-14.

Rodrigues, R. B. (2004). Avaliação e testagem psicológica no campo pericial. In L. C. I. Coronel (Ed.), Psiquiatria Legal: Informações cientificas para o leigo (pp. 148-150). Porto Alegre: Conceito.

Rovinski, S. (2007). Fundamentos da perícia psicológica forense. São Paulo: Vetor.

Santos, E. P. S. (2005). (Des)Construindo a 'menoridade': uma análise crítica sobre o papel da psicologia na produção da categoria “menor”. In H. S. Gonçalves \& E. P. Brandão (Eds.), Psicologia Jurídica no Brasil (pp. 205-248). Rio de Janeiro: Nau.

Saywitz, K. J., Mannarino, A. P., Berliner, L., \& Cohen, J. A. (2000). Treatment for sexually abused children and adolescents. American Psychologist, 55(9), 1040-1049.
Schreiber, E. (2001). Os direitos fundamentais da criança na violência intrafamiliar. Porto Alegre: Ricardo Lenz.

Shore, A. N. (2002). Dysregulation of the right brain: a fundamental mechanism of traumatic attachment and psychopathogenisis of posttraumatic stress disorder. Australian and New Zealand Journal of Psychiatry, 36(1), 9-30.

Silva, D. M. P. (2003). Psicologia Jurídica no Processo Civil Brasileiro. São Paulo: Casa do Psicólogo.

Silva Júnior, A. P. da (2006). Dano psíquico em crianças vitimas de abuso sexual sem comprovação de ato libidinoso ou conjunção carnal. Dissertação de Mestrado, Universidade de Brasília, Brasília.

Simão, R. B. C. (2007). Soluções judiciais concretas contra a perniciosa prática da alienação parental. In APASE (Ed.), Síndrome de Alienação Parental.

Stein, L. M., Pergher, G. K., \& Feix, L. da F. (2009). Desafios da oitiva de crianças no âmbito forense. Brasília-DF: Secretaria Especial dos Direitos Humanos da Presidência da República / Subsecretaria de Promoção dos Direitos da Criança e do Adolescente Programa Nacional de Enfrentamento da Violência Sexual contra Crianças e Adolescentes.

Steller, M., \& Boychuk, T. (1992). Children as witnesses in sexual abuse cases: investigative interview and assessment techniques. In H. Dent \& R. Flin (Eds.), Children as witnesses (pp. 47-73). New York, NJ: John Wiley \& Sons.

Trocmé, N., \& Bala, N. (2005). False allegations of abuse and neglect when parents separate. Child Abuse and Neglect, 29, 1333-1345.

Vrij, A. (2005). Criteria-based content analysis: A qualitative review of the first 37 studies. Psychology, Public Policy, and Law, 11, 3-41.

Welter, C. L. W., \& Feix, L. da F. (2010). Falsas memórias, sugestionabilidade e testemunho infantil. In L. M. Stein (Ed.), Falsas Memórias: fundamentos científicos e suas aplicações clínicas e jurídicas (pp. 157-185). Porto Alegre: Artmed.

Werner, J., \& Werner, M. C. M. (2008). Child sexual abuse in clinical and forensic psychiatry: a review of recent literature. Curr Opin Psychiatry, 21(5), 499-504.

World Health Organization. (1999). Report of the Consultation on Child Abuse Prevention. Geneva: World Health Organization.

World Health Organization. (2002). World report on violence and health. Geneva: World Health Organization.

World Health Organization. (2006). Preventing child maltreatment: a guide to taking action and generating evidence. Geneva: World Health Organization.

Ystgaard, M., Hestetun, I., Loeb, M., \& Mehlum, L. (2004). Is there a specific relationship between childhood sexual and physical abuse and repeated suicidal behavior? Child Abuse \& Neglect, 28(8), 863-875.

Recebido em 31.08.2010

Primeira decisão editorial em 28.01.2011

Versão final em 09.06.2011

Aceito em 13.01.2012 